\title{
UPAR expression under hypoxic conditions depends on iNOS modulated ERK phosphorylation in the MDA-MB-231 breast carcinoma cell line
}

So Young Yoon ${ }^{1}$, Yoo Jung Lee ${ }^{2}$, Jae Hong Seo ${ }^{3}$, Hwa Jung Sung ${ }^{3}$, Kyong Hwa Park ${ }^{3}$, In Keun Choi ${ }^{3}$, Seok Jin Kim³ Sang Cheul $\mathrm{Oh}^{3}$, Chul Won $\mathrm{Choi}^{3}$, Byung Soo Kim ${ }^{3}$, Sang Won Shin ${ }^{3}$, Yeul Hong Kim ${ }^{3}$, Jun Suk Kim ${ }^{3}$

${ }^{1}$ Division of Hematology/Oncology, Department of Internal Medicine, College of Medicine, Konkuk University, Seoul 143-729, Korea; ${ }^{2}$ Graduate School of Medicine, Korea University, Seoul 136-701, Korea $;{ }^{3}$ Division of Oncology, Department of Internal Medicine, College of Medicine, Korea University, Seoul 425-707, Korea

Urokinase plasminogen activator receptor ( $\mathrm{UPAR}$ ) plays a major role in cancer invasion and metastasis and uPAR expression is correlated with a poor prognosis in various cancer types. Moreover, the expression of uPAR is increased under hypoxic conditions. Nitric oxide (NO) and its metabolites produced by inducible nitric oxide synthase (iNOS) are important products of hypoxic stress, and NO may activate or modulate extracellular signal regulated kinase (ERK). Here, we evaluated UPA, uPAR, and activated ERK levels under hypoxic conditions, and the modulatory effects of iNOS and NO in the MDA-MB-231 human breast cancer cell line. Cells were incubated in a hypoxic or normoxic incubator and treated with PD98059 (a MEK 1/2 inhibitor, which abrogates ERK phosphorylation) and aminoguanidine (a selective iNOS inhibitor). uPAR expression, ERK phosphorylation, and uPA activity were found to be increased under hypoxic conditions. Moreover, when cells were treated with PD98059 under hypoxic conditions, uPAR was downregulated, whereas aminoguanidine markedly increased ERK phosphorylation in a dose dependent manner. Furthermore, aminoguanidine increased UPAR expression and prevented the inhibition of UPAR expression by PD98059. These results demonstrated that UPAR is induced by hypoxia and that increased UPAR expression is mediated by ERK phosphorylation, which in turn is modulated by iNOS/NO in MDA-MB-231 cells. We conclude that iNOS/NO downregulates the expression of uPAR under hypoxic conditions via ERK pathway modulation.

Cell Research (2006) 16:75-81. doi:10.1038/sj.cr.7310010; published online 16 January 2006

Keywords: uPAR, iNOS, ERK phosphorylation, hypoxia, MDA-MB-231

\section{Introduction}

Hypoxic stress underlies a number of important biological processes, such as, cellular migration and invasion,

Correspondence: Jae Hong Seo, Associate Professor of Division of Oncology \& Hematology, Department of Internal Medicine, College of Medicine, Korea University, Ansan Hospital 516 Kojan-dong, Dan Won-gu, Ansan city, Kyunggi-do, 425-707, Korea

Tel: 82-31-412-5886; Fax: 82-31-412-5806;

E-mail: cancer@korea.ac.kr

Received 1 Sep 2005; revised 8 Oct 2005; accepted 13 Oct 2005, published online 16 January 2006 and tumor growth [1-4]. For example, hypoxia within an expanding tumor leads to the release of vascular endothelial growth factor (VEGF) and the stimulation of angiogenesis, the success of which depends on endothelial cell migration and invasion [5, 6]. Hypoxia may promote cancer metastasis, which has been demonstrated in experimental tumor models [7-9]. However, the detailed mechanism by which hypoxia provokes cancer invasion and metastasis has not been fully elucidated.

Some tumor cell lines have been shown to up-regulate plasminogen activator inhibitor I (PAI-I) and UPAR when cells are exposed to hypoxia in vitro $[2-4,7,10,11]$. The uPAR gene promoter possesses HIF- $1 \alpha$ binding sites, and 
has been shown to be activated by HIF-1 $\alpha$ under hypoxic stress in human trophoblasts and in human umbilical vein endothelial cells [2]. In the normoxic state, uPA signaling through uPAR was observed to maintain an elevated basal level of activated ERK and to inhibit apoptosis in breast cancer cell lines [12]. Moreover, hypoxia induced the up-regulation of uPAR in a human prostate cancer cell line via ERK and p38 kinase signaling pathways [11]. The plasminogen activation system, leads to the formation of serine proteinase plasmin, and has been shown to play an important role in metastasis $[7,13,14]$. This activation system contains tissue plasminogen activator (tPA), uPA, PAI-1, PAI-2 and UPAR, and UPA is known to localize at the surface of tumor cells by binding to a specific receptor uPAR [15]. The uPAR/uPA complex is focused on the formation of plasmin and hence influences proteolytic activity in the vicinity of tumor cells. Plasmin facilitates tumor cell migration, invasion, and metastasis by degrading fibrin and other matrix proteins directly, and by activating several metalloproteinases that also degrade the extracellular matrix [16].

Nitric oxide (NO) and its metabolites, which are produced by nitric oxide synthases (NOSs), are important products of hypoxic stress [17-19]. And, human breast carcinoma cells and mouse mammary tumor cell lines produce NO in amounts correlated with tumor grade and metastasis [20-22]. Moreover, breast cancer patients with an iNOS positive tumor have a poorer outcome than those with an iNOS negative tumor [23]. The mechanisms by which NO may enhance mammary tumor development and metastases include increasing DNA damage and tumor cells migration, and promoting angiogenesis [21, 24]. NO may activate or modulate MAPK/ERK, G-proteins, the Ras pathway and PI3K signaling [21, 24, 25]. However, the molecular interactions between iNOS/NO, ERK, and UPAR in hypoxic states have not been elucidated. In this study, we examined uPAR expression and ERK activation in human MDA-MB-231 breast cancer cells under hypoxic conditions and investigated the roles of ERK, iNOS/NO as upstream regulators of UPAR.

\section{Material and methods}

\section{Cell lines and hypoxic culture conditions}

The human metastatic breast carcinoma cell line, MDA-MB231 was used throughout this study. Cells were purchased from KCLB (the Korean Cell Line Bank, Seoul) and cultured in RPMI 1640 medium supplemented with $10 \%$ fetal bovine serum (FBS), $100 \mathrm{units} / \mathrm{ml}$ penicillin and $100 \mu \mathrm{g} / \mathrm{ml}$ streptomycin in a humidified atmosphere of $5 \% \mathrm{CO}_{2}$ and $95 \%$ air at $37{ }^{\circ} \mathrm{C}$. All cells were used at $80 \%$ confluence. For cell culture under $1 \% \mathrm{O}_{2}$ tension the cells were incubated for $2 \mathrm{~h}$ to $48 \mathrm{~h}$ in a humidified atmosphere at $37{ }^{\circ} \mathrm{C}$ in a multi-gas $\mathrm{CO}_{2}-\mathrm{O}_{2}$ incubator (NUAIRE, Plymouth, $\mathrm{MN}$ ) equilibrated with $1 \% \mathrm{O}_{2}, 5 \% \mathrm{CO}_{2}$, and $94 \% \mathrm{~N}_{2}$. Control cultures were performed in $20 \% \mathrm{O}_{2}$ and $5 \% \mathrm{CO}_{2}$, in a $\mathrm{CO}_{2}$ incubator (NUAIRE, Plymouth, $\mathrm{MN}$ ) at $37^{\circ} \mathrm{C}$. Cell harvesting was performed in a hypoxic chamber.

\section{Gel zymography and $u P A$ activity}

uPA enzyme activity in culture media was measured by gel zymography. $1 \times 10^{6}$ cells were seeded in $10 \mathrm{~cm}^{2}$ dishes and when sub-confluent, media were changed to serum free media to exclude the influence of serum factors. After a $3 \mathrm{~h}$ equilibration period, the cells were incubated in the hypoxic incubator. Media were collected and concentrated with a serum concentrating kit after $4 \mathrm{~h}, 8 \mathrm{~h}$, and $12 \mathrm{~h}$ of hypoxia treatment. A sample volume containing $30 \mu \mathrm{g}$ of protein was mixed with SDS sample buffer and loaded into an SDS/polyacrylamide gel containing $2 \mathrm{mg} / \mathrm{ml} \alpha$-casein and 0.025 units $/ \mathrm{ml}$ of plasminogen (Sigma, St. Louis, MO). Following electrophoresis, gels were washed twice for $10 \mathrm{~min}$ with $2.5 \%$ Triton X-100 (Sigma, St. Louis, MO) in water, rinsed briefly with water, and incubated overnight in a solution of $50 \mathrm{mM}$ TRIS and $5 \mathrm{mM} \mathrm{CaCl}$. The gels were then stained with $0.4 \%$ Coomassie brilliant blue R-250 in $10 \%$ acetic acid $/ 40 \%$ methanol, destained in $10 \%$ acetic acid $/ 40 \%$ methanol, and dried between sheets of cellophane on a gel dryer (Vision, Bucheon, Korea).

\section{Western blot analysis for $u P A R, E R K, H I F-1 \alpha$ expression}

Cells were washed in PBS, detached using Trypsin-EDTA buffer, and stored at $-70{ }^{\circ} \mathrm{C}$. Protein was extracted with RIPA buffer $(1 \%$ NP-40/Ig PAL, $0.5 \%$ sodium deoxycholate, $0.1 \%$ SDS) and protease inhibitors (aprotinin, leupeptin, PMSF, benzamidine, trypsin inhibitor, sodium orthovanadate). Total protein was analyzed quantitatively using a spectrophotometer at $595 \mathrm{~nm} .30 \mu \mathrm{g}$ of proteins were then separated by SDS-PAGE and transferred to polyvinylidene fluoride membranes. Membranes were incubated with anti-uPAR goat polyclonal antibody, and phosphorylated ERK (p-ERK) polyclonal antibody (Santa Cruz Biotechnology, Santa Cruz, CA), and bound antibody was detected by biotin-streptavidin alkaline phosphatase staining followed by ECL (Amersham, Piscataway, NJ). After 3 min to $30 \mathrm{~min}$, films were developed and analyzed. Blots were probed with anti- $\beta$-actin mouse monoclonal antibody (Sigma Chemical Co., St. Louis, MO) as a loading control. Protein molecular weights were estimated using prestained standards, according the manufacturer's instructions.

\section{Drug treatments}

To evaluate the contributions of the ERK pathway and NO signaling, we used an MEK 1/2 inhibitor (PD 98059, Sigma, St. Louis, MO), which abrogates ERK phosphorylation, and a selective iNOS inhibitor (aminoguanidine). All drugs were purchased from Sigma (St. Louis, MO)

\section{Results}

Hypoxia induced uPAR expression in MDA-MB-231 cells via ERK signaling

To determine the time course of UPAR expression and ERK activation in MDA-MB-231 cells under hypoxic conditions, cells were cultured in the $1 \% \mathrm{O}_{2}$ atmosphere for various times. As expected, the induction of uPAR protein was detected as early as $4 \mathrm{~h}$ after exposure to hypoxia and 


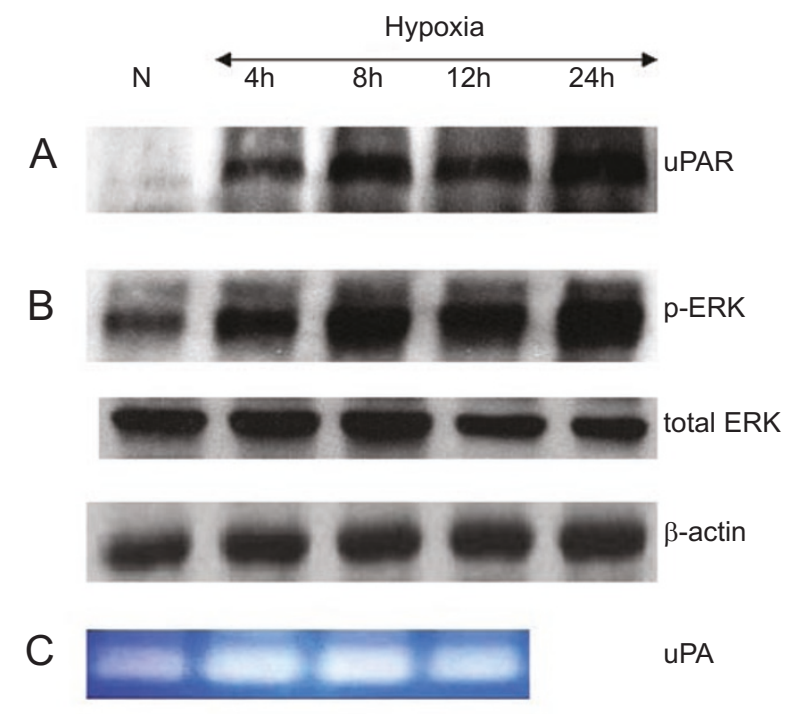

Figure 1 Induction of the uPA/uPAR signal and the phosphorylation of ERK in human MDA-MB-231 cells under hypoxia. (A) uPAR was markedly induced in a $1 \% \mathrm{O}_{2}$ environment after 4 to $24 \mathrm{~h}$. (B) ERK phosphorylation was induced after exposure to hypoxia for the indicated times. (C) uPA activity by gel zymography was increased after 4,8 and $12 \mathrm{~h}$ of culture in a hypoxic environment. The data shown represent three separate experiments. N: normoxia.

remained elevated for $24 \mathrm{~h}$ (Figure 1). In two other time course experiments, we found that the maximal level of uPAR induction was sustained for $48 \mathrm{~h}$ (data not shown). To determine whether hypoxia induces ERK phosphorylation in MDA MB 231 cells, we examined ERK phosphorylation at various times following exposure to $1 \%$ hypoxia. As was observed for uPAR, phosphorylated ERK levels were elevated under hypoxic conditions (Figure 1B). The induction of UPA activity began at $4 \mathrm{~h}$ after exposure to $1 \%$ hypoxia and remained until $12 \mathrm{~h}$ in MDA-MB-231 cells by gel zymography, though the degree of induction was modest (Figure 1C). Taken together, both p-ERK and uPA/uPAR signals were induced following the exposure of MDA-MB-231 human breast cancer cells to $1 \%$ hypoxia. In human epidermoid carcinoma cell lines and human colon carcinoma cell lines, agents that regulate ERK activation were also found to regulate the expressions of UPA and uPAR $[26,27]$. The ERK pathway is activated after exposure to hypoxia $[28,29]$, and thus, ERK activation may affect cell invasion and metastasis indirectly by affecting the expressions of UPA and UPAR. To determine whether uPAR expression is dependent on ERK phosphorylation in hypoxic conditions, MDA-MB-231 cells were treated with the MEK1/2 inhibitor PD98059 under 1\% hypoxia, and it was found that uPAR expression was markedly decreased
A

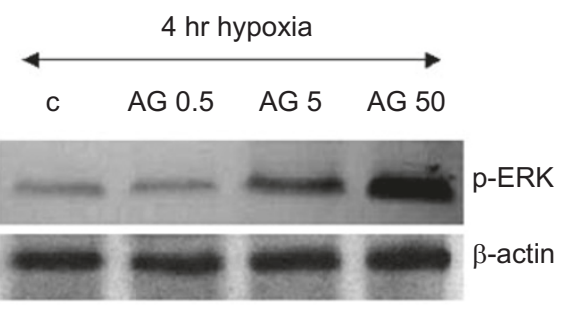

B

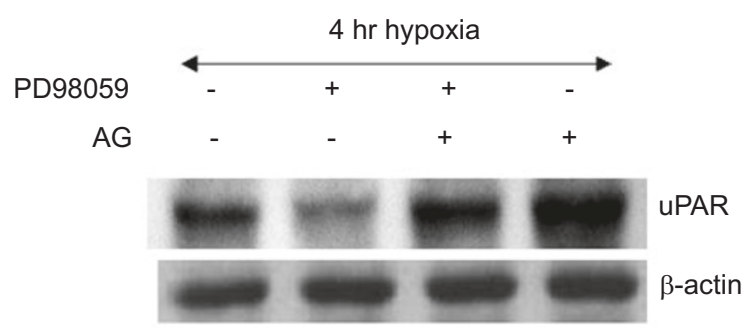

Figure 2 Modulation of uPAR expression by ERK and iNOS/NO in MDA-MB-231 human breast cancer cells under hypoxia. (A) Aminoguanidine, a selective iNOS inhibitor, increased ERK phosphorylation in a dose dependent manner at concentrations of $0.5,5$, and $50 \mu \mathrm{M}$ in MDA-MB-231 cells exposed to hypoxia for $4 \mathrm{~h}$. ERK phosphorylation was dramatically increased by the selective iNOS inhibitor, aminoguanidine. (B) The MEK 1/2 inhibitor, PD98059 (20 $\mu \mathrm{M})$ prevented uPAR induction in human MDA-MB-231 breast cancer cells exposed to hypoxia for $4 \mathrm{~h}$ (lane 2). $5 \mu \mathrm{M}$ of aminoguanidine prevented the inhibition of UPAR by PD 98059 and increased uPAR expression in MDA-MB231 cells under hypoxic stress $\left(3^{\text {rd }}\right.$ and $4^{\text {th }}$ lanes). The data shown represent three separate experiments. AG: aminoguanidine. Blotting with anti $\beta$-actin antibody showed that lanes were equally loaded.

(Figure 2B). These data provide evidence that uPAR expression in MDA-MB-231 cells under hypoxic conditions depends on the activation of ERK.

The selective iNOS inhibitor, aminoguanidine, increased uPAR expression by modulating the ERK pathway

To test whether iNOS/NO modulates ERK phosphorylation in MDA-MB-231 cells under hypoxic conditions, cultures were treated with aminoguanidine under $1 \%$ hypoxic conditions for $4 \mathrm{~h}$. As shown Figure 2A, aminoguanidine induced ERK phosphorylation in a dose dependent manner in MDA-MB-231 cells. We hypothesized that inhibiting iNOS would upregulate hypoxia-induced uPAR expression via the ERK pathway. As was expected, aminoguanidine prevented the inhibition of UPAR by PD98059 ( $3^{\text {rd }}$ lane) and increased uPAR expression ( $4^{\text {th }}$ lane). Taken together these results suggest that NO decreases uPAR expression by inhibiting ERK dependent and independent pathways. 


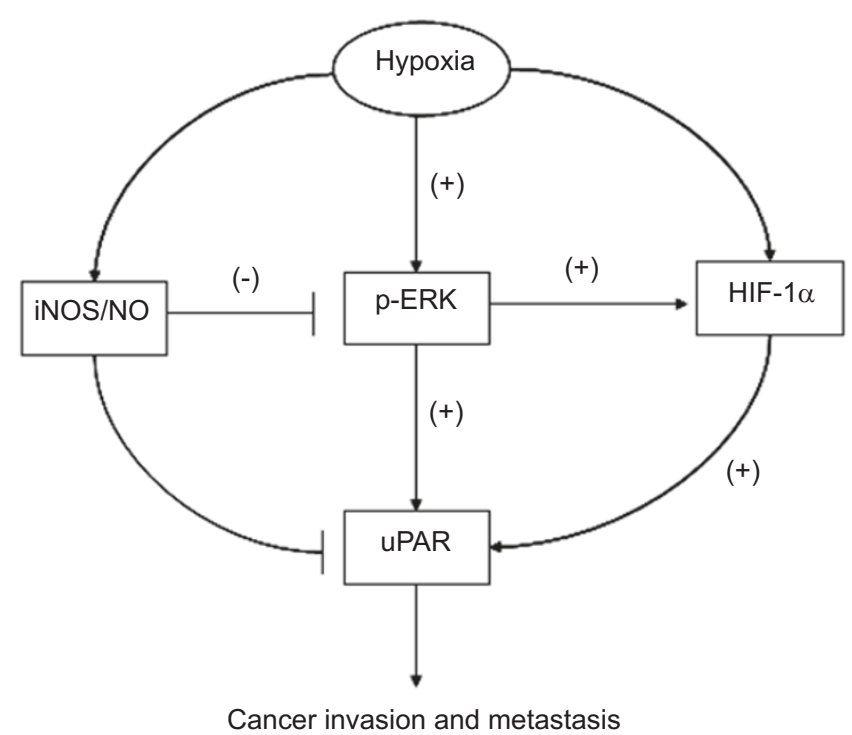

Figure 3 Schematic diagram of the uPA-uPAR regulatory system. It is well known that hypoxia increases the invasion and migration of malignant tumor cells by activating $\mathrm{UPA} / \mathrm{uPAR}$ signaling via HIF-1 $\alpha$ transcription $[2-4,7,34]$. NO and its metabolites produced by iNOS are important products of hypoxic stress [17-20], and ERK activation is known to be needed for HIF-1 $\alpha$ stabilization [28, 29, 45] and HIF-1 $\alpha$ activation [29]. In the present study shows that uPA/uPAR signaling was modulated by the ERK and iNOS/NO pathways in human MDA-MB-231 cells under hypoxic stress. Hypoxia induced uPAR expression in human MDA-MB-231 breast cancer cells, and uPAR expression under hypoxia in MDA-MB-231 cells depends on the phosphorylation of ERK. iNOS/NO signaling blocks uPAR expression in MDA-MB-231 cells via ERK dependent and independent pathways. The effect of $\mathrm{iNOS} / \mathrm{NO}$ on HIF-1 $\alpha$ is controversial [18-20, 34, 43].

\section{Discussion}

The major finding of the present study is that uPAR induction in MDA-MB-231 human breast cancer cells under hypoxia depends on the ERK signaling pathway, and which is regulated by the iNOS/NO pathway. Moreover, iNOS and NO had an effect on UPAR induction under hypoxia through both an ERK dependent and an ERK independent pathway (Figure 3).

Many studies have shown that hypoxia increases cancer cell invasion and metastasis in various tumor types via the upregulation of the uPA/uPAR signal transduction pathway $[2-4,7,34]$. The uPAR gene promoter is known to possess three HIF- $1 \alpha$ binding sites and to be activated by HIF-1 $\alpha$ under hypoxic stress [2]. However, the roles of ERK and iNOS/NO signaling in the modulation of UPAR are not well understood.

The roles that NO plays in various tumors remain con- troversial and prompts the question "friend or foe". Some reports have found that NO is cytotoxic [35-37], whilst others have shown that it possesses protective properties against reactive oxygen species (ROS) [38, 39]. Thus, NO probably plays a dual role that depends on its intratumoral concentration and exposure duration. Patients with iNOS positive breast carcinomas were found to have significantly poorer overall survival rates than those with iNOS negative tumors [23]. Moreover, in iNOS knock out mice mammary gland tumor latency was found to be increased, suggesting that $\mathrm{NO}$ production in vivo promotes mammary tumor formation [31]. In other models, NO was found to promote murine mammary gland tumor growth and metastasis by stimulating tumor cell migration, invasiveness and angiogenesis $[22,30]$, which are processes that require the sequential activations of nitric oxide synthase, guanylate cyclase and mitogen-activated protein kinase [24]. However, those studies were done in normoxic states, and it was suggested that iNOS and NO have different roles in tumor invasion and metastasis and that these are determined by oxygen tension [40, 41]. Moreover, most tumors develop central necrosis and hypoxic regions if they grow beyond a certain size due to inadequate vascularization [1]. Study in the hypoxic state may reflect the physiologic condition of cancer. The present study utilized human MDA-MB-231 breast cancer cells under hypoxic conditions, and showed that protective roles of iNOS/NO system in human MDAMB-231 breast cancer cells are via the down-regulation of ERK expression and consequently the inhibition of UPAR induction.

cGMP and PKG are well known second messengers iNOS/NO [24, 33, 40, 42], as may be cytochrome C oxidase in cellular mitochondria $[38,43]$. Graham et al. studied the influence of NO on tumor cell invasiveness in MDA-MB231 cells under hypoxic states [3], and showed that NO reduced UPAR induction under hypoxic states in a cGMP dependent manner. However, the manner in which cGMP reduces UPAR signaling under hypoxic states has not been defined. Our study is the first study to find that iNOS/NO inhibits uPAR induction via an ERK dependent pathway in human MDA-MB-231 breast cancer cells exposed to hypoxic states, which might reduce the invasiveness and metastatic potential of breast cancer. Other studies have also found that the ERK pathway is activated under hypoxic stress [28, 29]. Phosphorylation of ERK was reported to be more extensive in hypoxic human prostate cancer cells than in normoxic cells [11]. A study also showed that NO inhibited the phosphorylation of ERK via cGMP mediated interference of the ras/raf pathway [44], and another that NO and cGMP mimetic drugs inhibited elastase activity in vascular smooth muscle cells [42].

The present study also shows that iNOS/NO suppressed 
UPAR activity in an ERK independent manner, since aminoguanidine decreased uPAR expression even in the presence of PD98059 in MDA-MB-231 cells under hypoxic stress. ROS like superoxide and hydroxyperoxynitrite are increased during hypoxia [45], and ROS have also been found to increase tumorigenesis and tumor invasion and metastasis $[39,46]$. The iNOS/NO signal competes with oxygen to bind cytochrome c oxidase, which could decrease ROS generation [38].

A key objective of our research is to understand changes in $\mathrm{UPA}$ and $\mathrm{UPAR}$ expression and their regulation under hypoxic conditions, since the majority of cancers grow in such an environment [1, 7-9]. In the present study, uPAR expression was increased under hypoxic stress, which concurs with the study of Graham et al. which showed that hypoxia stimulates carcinoma cell invasiveness via the upregulation of urokinase receptor expression [2, 3]. In our study, uPA activity (as measured by gel zymography) was modestly induced in hypoxic conditions, and UPAR was markedly induced, which suggests that an up-regulated uPA-uPAR system in hypoxia is due to both UPAR upregulation and increased uPA activity.

In endothelial cells of the porcine aorta, hypoxia activated ERK and $\mathrm{NAD}(\mathrm{P}) \mathrm{H}$ oxidase, and triggered a burst of ROS [45], and the UPA-uPAR system was also found to influence the activation of ERK, such that ERK and the uPA-uPAR system formed a positive feedback loop [8, 26, 27]. Moreover, ERK has been reported to be a key regulator of tumor cell proliferation, invasion and metastasis via the ROS pathway and matrix metalloproteinase $[47,48]$ and the ERK pathway was found to be activated upon exposure to hypoxia [28, 29, 45]. Moreover, hypoxia induced uPAR up-regulation was inhibited by the specific MEK 1/2 inhibitor, PD98059, in human prostate cancer cells [11].

These findings caused us to believe that ERK activation could be a main regulator of the uPAR system in the MDA-MB-231 human breast cancer cell line under hypoxic stress. Indeed, ERK phosphorylation was upregulated under hypoxia, and PD98059 markedly decreased uPAR expression. These results show that hypoxia can induce uPAR through ERK activation. Our results demonstrate for the first time that under hypoxic conditions the ERK signaling pathway is an upstream regulator of the UPAR system in human MDA-MB-231 breast cancer cells. In addition, ERK phosphorylation is known to be required both for HIF- $1 \alpha$ stabilization $[28,29,45]$ and the HIF- $1 \alpha$ activation of transcriptional activity [29]. Therefore, it is possible that HIF- $1 \alpha$ has both an indirect influence on the UPA-uPAR system via the ERK signaling pathway and a direct influence on UPAR by binding its transcription region.

We suggest that the activation of ERK could result in uncontrolled tumor proliferation and invasion via the uPA-
uPAR system in human MDA-MB-231 breast cancer cells under hypoxic stress. NO and iNOS have an inhibitory effect on the uPA-uPAR system by inhibiting ERK phosphorylation in MDA-MB-231 breast cancer cells exposed to hypoxic stress. Conclusively, our data provide evidence; 1) that hypoxia-induced UPAR expression in human MDA-MB-231 cells depends on ERK phosphorylation; 2) that iNOS/NO signaling inhibits uPAR induction under hypoxic conditions via ERK dependent and independent pathways.

\section{References}

1 Harris AL. Hypoxia - a key regulatory factor in tumor growth. Nat Rev Cancer 2002; 2:38-47.

2 Graham CH, Fitzpatrick TE, McCrae KR. Hypoxia stimulates urokinase receptor expression through a heme protein-dependent pathway. Blood 1998; 91:3300-7.

3 Graham CH, Forsdike J, Fitzgerald CJ, Macdonald-Goodfellow S. Hypoxia-mediated stimulation of carcinoma cell invasiveness via upregulation of urokinase receptor expression. Int J Cancer 1999; 80:617-23.

4 Krishnamachary B, Berg-Dixon S, Kelly B, et al. Regulation of colon carcinoma cell invasion by hypoxia-inducible factor 1 . Cancer Res 2003; 63:1138-43.

5 Fukuda R, Hirota K, Fan F, et al. Insulin-like growth factor 1 induces hypoxia-inducible factor 1-mediated vascular endothelial growth factor expression, which is dependent on MAP kinase and phosphatidylinositol 3-kinase signaling in colon cancer cells. J Biol Chem 2002; 277:38205-11.

6 Rofstad EK, Danielsen T. Hypoxia-induced metastasis of human melanoma cells: involvement of vascular endothelial growth factor-mediated angiogenesis. Br J Cancer 1999; 80:1697-707.

7 Rofstad EK, Rasmussen H, Galappathi K, et al. Hypoxia promotes lymph node metastasis in human melanoma xenografts by up-regulating the urokinase-type plasminogen activator receptor. Cancer Res 2002; 62:1847-53.

8 De Jaeger K, Kavanagh MC, Hill RP. Relationship of hypoxia to metastatic ability in rodent tumours. Br J Cancer 2001; 84:12805.

9 Young SD, Marshall RS, Hill RP. Hypoxia induces DNA overreplication and enhances metastatic potential of murine tumor cells. Proc Natl Acad Sci U S A 1988; 85:9533-7.

10 Koong AC, Denko NC, Hudson KM, et al. Candidate genes for the hypoxic tumor phenotype. Cancer Res 2000; 60:883-7.

11 Lee KH, Choi EY, Hyun MS, Kim JR. Involvement of MAPK pathway in hypoxia-induced up-regulation of urokinase plasminogen activator receptor in a human prostatic cancer cell line, PC3MLN4. Exp Mol Med 2004; 36:57-64.

12 Ma Z, Webb DJ, Jo M, Gonias SL. Endogenously produced urokinase-type plasminogen activator is a major determinant of the basal level of activated ERK/MAP kinase and prevents apoptosis in MDA-MB-231 breast cancer cells. J Cell Sci 2001; 114:3387-96.

13 de Vries TJ, van Muijen GN, Ruiter DJ. The plasminogen activation system in tumour invasion and metastasis. Pathol Res Pract 1996; 192:718-33. 
14 Blasi F, Carmeliet P. uPAR: a versatile signaling orchestrator. Nat Rev Mol Cell Biol 2002; 3:932-43.

15 Schmitt M, Harbeck N, Thomssen C, et al. Clinical impact of the plasminogen activation system in tumor invasion and metastasis: prognostic relevance and target for therapy. Thromb Haemost 1997; 78:285-96.

16 Pollanen J, Stephens RW, Vaheri A. Directed plasminogen activation at the surface of normal and malignant cells. Adv Cancer Res 1991; 57:273-328.

17 Melillo G, Musso T, Sica A, et al. A hypoxia-responsive element mediates a novel pathway of activation of the inducible nitric oxide synthase promoter. J Exp Med 1995; 182:1683-93.

18 Yu L, Gengaro PE, Niederberger M, et al. Nitric oxide: a mediator in rat tubular hypoxia/reoxygenation injury. Proc Natl Acad Sci U S A 1994; 91:1691-5.

19 Melillo G, Taylor LS, Brooks A, et al. Functional Requirement of the Hypoxia-responsive Element in the Activation of the Inducible Nitric Oxide Synthase Promoter by the Iron Chelator Desferrioxamine. J Biol Chem 1997; 272:12236-43.

20 Duenas-Gonzalez A, Isales CM, del Mar Abad-Hernandez M, et al. Expression of inducible nitric oxide synthase in breast cancer correlates with metastatic disease. Mod Pathol 1997; 10:645-9.

21 Phoa N, Epe B. Influence of nitric oxide on the generation and repair of oxidative DNA damage in mammalian cells. Carcinogenesis 2002; 23:469-75.

22 Vakkala M, Kahlos K, Lakari E, et al. Inducible nitric oxide synthase expression, apoptosis, and angiogenesis in in situ and invasive breast carcinomas. Clin Cancer Res 2000; 6:2408-16.

23 Loibl S, Buck A, Strank C, et al. The role of early expression of inducible nitric oxide synthase in human breast cancer. European Journal of Cancer 2005; 41:265-71.

24 Jadeski LC, Chakraborty C, Lala PK. Nitric oxide-mediated promotion of mammary tumour cell migration requires sequential activation of nitric oxide synthase, guanylate cyclase and mitogen-activated protein kinase. Int J Cancer 2003; 106:496-504.

25 Chan ED, Riches DWH. IFN- $\gamma+$ LPS induction of iNOS is modulated by ERK, JNK/SAPK, and p38mapk in a mouse macrophage cell line. Am J Physiol Cell Physiol 2001; 280: C441-50.

26 Ahmed N, Oliva K, Wang Y, et al. Proteomic profiling of proteins associated with urokinase plasminogen activator receptor in a colon cancer cell line using an antisense approach. Proteomics 2003; 3:288-98.

27 Aguirre Ghiso JA, Kovalski K, Ossowski L. Tumor Dormancy Induced by Downregulation of Urokinase Receptor in Human Carcinoma Involves Integrin and MAPK Signaling. J Cell Biol 1999; 147:89-104.

28 Minet E, Arnould T, Michel G, et al. ERK activation upon hypoxia: involvement in HIF-1 activation. FEBS Letters 2000; 468:53-8.

29 Mottet D, Michel G, Renard P, et al. Role of ERK and calcium in the hypoxia-induced activation of HIF-1. J Cell Physiol 2003; 194:30-44.

30 Jadeski LC, Hum KO, Chakraborty C, Lala PK. Nitric oxide promotes murine mammary tumour growth and metastasis by stimulating tumour cell migration, invasiveness and angiogenesis. Int J Cancer 2000; 86:30-9.

31 Ellies LG, Fishman M, Hardison J, et al. Mammary tumor latency is increased in mice lacking the inducible nitric oxide synthase. Int J Cancer 2003; 106:1-7.

32 Palmer LA, Semenza GL, Stoler MH, Johns RA. Hypoxia induces type II NOS gene expression in pulmonary artery endothelial cells via HIF-1. Am J Physiol Lung Cell Mol Physiol 1998; 274: L212-9.

33 Nagao K, Takenaka S, Yamaji R, et al. Nitric oxide synthase induction, cGMP elevation, and biopterin synthesis in vascular smooth muscle cells stimulated with interleukin-1â in hypoxia. J Biochem (Tokyo) 2003; 133:501-5.

34 Rofstad EK, Mathiesen B, Galappathi K. Increased metastatic dissemination in human melanoma xenografts after subcurative radiation treatment: radiation-induced increase in fraction of hypoxic cells and hypoxia-induced up-regulation of urokinase-type plasminogen activator receptor. Cancer Res 2004; 64:13-8.

35 Radi R, Beckman J, Bush K, Freeman B. Peroxynitrite oxidation of sulfhydryls. The cytotoxic potential of superoxide and nitric oxide. J Biol Chem 1991; 266:4244-50.

36 Noronha-Dutra AA, Epperlein MM, Woolf N. Reaction of nitric oxide with hydrogen peroxide to produce potentially cytotoxic singlet oxygen as a model for nitric oxide-mediated killing. FEBS Lett 1993; 321:59-62.

37 Polte T, Oberle S, Schroder H. Nitric oxide protects endothelial cells from tumor necrosis factor-alpha-mediated cytotoxicity: possible involvement of cyclic GMP. FEBS Lett 1997; 409:468.

38 Palacios-Callender M, Quintero M, Hollis VS, et al. Endogenous NO regulates superoxide production at low oxygen concentrations by modifying the redox state of cytochrome c oxidase. Proc Natl Acad Sci U S A 2004; 101:7630-5.

39 Wink D, Hanbauer I, Krishna M, et al. Nitric Oxide Protects Against Cellular Damage and Cytotoxicity From Reactive Oxygen Species. Proc Natl Acad Sci U S A 1993; 90:9813-7.

40 Postovit LM, Adams MA, Lash GE, et al. Oxygen-mediated regulation of tumor cell invasiveness. Involvement of a nitric oxide signaling pathway. J Biol Chem 2002; 277:35730-7.

41 Matthews NE, Adams MA, Maxwell LR, et al. Nitric OxideMediated Regulation of Chemosensitivity in Cancer Cells. J Natl Cancer Inst 2001; 93:1879-85.

42 Mitani Y, Zaidi SHE, Dufourcq P, et al. Nitric oxide reduces vascular smooth muscle cell elastase activity through cGMPmediated suppression of ERK phosphorylation and AML1B nuclear partitioning. FASEB J 2000; 14:805-14.

43 Mateo J, Garcia-Lecea M, Cadenas S, et al. Regulation of hypoxia-inducible factor-1 alpha by nitric oxide through mitochondria-dependent and -independent pathways. Biochem J 2003; 376:537-44.

44 Yu SM, Hung LM, Lin CC. cGMP-elevating agents suppress proliferation of vascular smooth muscle cells by inhibiting the activation of epidermal growth factor signaling pathway. Circulation 1997; 95:1269-77.

45 Schafer M, Schafer C, Ewald N, et al. Role of redox signaling in the autonomous proliferative response of endothelial cells to hypoxia. Circ Res 2003; 92:1010-5.

46 Zhang HJ, Zhao W, Venkataraman S, et al. Activation of matrix netalloproteinase- 2 by overexpression of manganese superoxide dismutase in human rreast cancer MCF-7 cells involves reactive oxygen species. J Biol Chem 2002; 277:20919-26.

47 Johansson N, Ala-aho R, Uitto V, et al. Expression of collage- 
nase-3 (MMP-13) and collagenase-1 (MMP-1) by transformed keratinocytes is dependent on the activity of $\mathrm{p} 38$ mitogen-activated protein kinase. J Cell Sci 2000; 113 Pt 2:227-35.
$48 \mathrm{Kim}$ MS, Lee EJ, Kim HR, Moon A. p38 kinase is a key signaling molecule for H-Ras-induced cell motility and invasive phenotype in human breast epithelial cells. Cancer Res 2003; 63:5454-61. Edited by Zhong Cheng Zheng 